\title{
Nonlinear dynamics of frontal polymerization with autoacceleration
}

\author{
D.M.G. COMISSIONG, L.K. GROSS ${ }^{1}$ and V.A. VOLPERT* \\ Department of Engineering Sciences and Applied Mathematics, Northwestern University, Evanston, IL 60208-3125, \\ USA; ${ }^{1}$ Department of Theoretical and Applied Mathematics, The University of Akron, Akron, OH 44325-4002, \\ USA; *Author for correspondence (v-volpert@northwestern.edu)
}

Received 15 June 2004; accepted in revised form 17 February 2005

\begin{abstract}
A free-boundary model is used to describe frontal polymerization. Autoacceleration effects, such as the gel effect, are accounted for in the formulation. Weakly nonlinear analysis is applied to investigate pulsating instabilities in two dimensions. The analysis produces a pair of Landau equations that describe the evolution of the linearly unstable modes. Autoacceleration influences the linear stability of the system as well as the onset and stability of spinning and standing modes.
\end{abstract}

Key words: frontal polymerization, Landau equations, nonlinear stability

\section{Introduction}

We consider the nonlinear dynamics of a free-radical polymerization front in two dimensions. Frontal polymerization (FP) was first documented experimentally in [1]. It is the process by which conversion from monomer to polymer occurs in a narrow region that propagates in space. In the simplest case, a polymerization front can be generated in a test tube containing a mixture of monomer and initiator by supplying heat to one end of the tube. The heat subsequently decomposes the initiator into free radicals which trigger the highly exothermic process of free-radical polymerization. The heat released by the reaction diffuses ahead, increases the temperature there causing initiator decomposition, and the process repeats. The focus of our attention will be the self-sustaining wave which travels through the tube as polymer molecules are being formed. Uniformly propagating planar waves may become unstable as parameters vary resulting in interesting nonlinear behaviors [2]. A clear understanding of the stability of the propagating front is necessary to achieve both the desired uniformity and the overall quality of the product. A complete linear stability analysis was first presented by Schult and Volpert [3] and Spade and Volpert [4]. Weakly nonlinear analyses for the simplest model were performed in $[5,6]$. The focus of this paper is a weakly nonlinear analysis of a more complex model that allows for autoacceleration of the polymerization reactions by the reaction product. There may be various reasons for such autoacceleration. One possibility is the gel effect. A simplified description of the gel effect is as follows. Theoretically, the polymerization rate should decrease with a depletion of the available supply of the monomer and initiator. Based on this fact alone the rate of conversion to the polymer state should decrease with time. However, it has been experimentally verified that up to a certain stage, the overall polymerization rate may decrease, but beyond this point it increases. This anomaly is referred to as the gel effect. An explanation for this phenomenon is the rapid growth of polymer chains, which make the mixture more viscous so that longer chains can become entangled more easily. 
This decreases the termination rate thus increasing the overall polymerization rate. Another example of autoacceleration occurs in case of polymerization of multi-functional monomers. Kinetic measurements indicate that the polymerization rate increases with the degree of conversion.

The paper is structured as follows. First, we discuss the chemical kinetics of the free-radical polymerization process, and formulate a mathematical model based on mass and heat balances in the system. Then we use several simplifications to reduce the problem to a moving-free-boundary problem. We determine uniformly propagating one-dimensional traveling waves, and perform their linear stability analysis. Weakly nonlinear stability analysis results in a pair of Landau equations, the solutions of which are discussed depending on the parameters of the problem.

\section{Mathematical model}

Most frontal polymerization studies involve free-radical polymerization kinetics. The freeradical polymerization in question consists of a standard sequence of chemical reactions [7, Chapter 3]. In particular, when one end of the tube is heated, the initiator $\widetilde{I}$ decomposes, forming two radicals $\widetilde{R}$. Each radical can then combine with a monomer, initiating a polymer chain $\widetilde{P}_{1}^{*}$. Here $\widetilde{P}_{1}^{*}$ is also a radical, and the subscript implies that it contains one monomer unit. The proportion $f$ of free radicals $\widetilde{R}$ used in the initiation step is called an efficiency factor and is initiator-dependent. A polymer chain $\widetilde{P}_{n}^{*}, n=1,2, \ldots$ grows by combining with another monomer to form the chain $\widetilde{P}_{n+1}^{*}$, and terminates by combining with a radical, either another growing chain $\widetilde{P}_{m}^{*}$ or an initiator radical $\widetilde{R}$. The kinetic scheme that involves the decomposition step, initiation step, propagation step and the two possible termination steps can be summarized as

$$
\begin{aligned}
& \widetilde{I} \stackrel{k_{d}}{\rightarrow} f \times 2 \widetilde{R} \quad \text { (initiator decomposition) } \\
& \widetilde{R}+\widetilde{M} \stackrel{k_{p}}{\rightarrow} \widetilde{P}_{1}^{*} \quad \text { (chain initiation) } \\
& \widetilde{P}_{n}^{*}+\widetilde{M} \stackrel{k_{p}}{\rightarrow} \widetilde{P}_{n+1}^{*} \quad \text { (chain growth, also known as chain propagation) } \\
& \widetilde{P}_{n}^{*}+\widetilde{P}_{m}^{*} \stackrel{k_{t}}{\rightarrow} \widetilde{P} \quad \text { (polymer radical termination) } \\
& \widetilde{P}_{n}^{*}+\widetilde{R} \stackrel{k_{t}}{\rightarrow} \widetilde{P} \quad \text { (primary radical termination) }
\end{aligned}
$$

Here $\widetilde{I}, \widetilde{R}, \widetilde{M}, \widetilde{P}$ represent initiator, primary radical, monomer and chemically unreactive polymer molecule, respectively. Next, $\widetilde{P}_{n}^{*}, n=1,2, \ldots$ represents a polymer radical with $n$ monomer units, $f$ is an efficiency factor, which is dependent on the initiator used [7, pp. 232-240]. The reaction rate parameters denoted as $k$ with a subscript depend on temperature and have the form of Arrhenius exponentials

$$
\begin{aligned}
k_{d} & =k_{d}^{0} \exp \left\{-E_{d} /\left(R_{g} T\right)\right\}, \quad k_{p}=k_{p}^{0} \exp \left\{-E_{p} /\left(R_{g} T\right)\right\}, \\
k_{t} & =k_{t}^{0} \exp \left\{-E_{t} /\left(R_{g} T\right)\right\},
\end{aligned}
$$

where $R_{g}$ and $T$ are the gas constant and temperature, $k_{d}^{0}, k_{p}^{0}, k_{t}^{0}$ and $E_{d}, E_{p}, E_{t}$ are the frequency factors and activation energies of decomposition, propagation and termination reactions. The activation energies are assumed to be constant. The frequency factors, however, may be functions of the monomer concentration $M$. Specifically, we assume that $k_{p}^{0}$ and $k_{t}^{0}$ depend on $M$, while $k_{d}^{0}$ is constant. The dependence of $k_{t}^{0}$ on $M$ models the gel effect; $k_{t}^{0}(M)$ is an increasing function, which is equivalent to saying that the termination rate decreases 
with an increase in the degree of conversion. The dependence of $k_{p}^{0}$ on $M$ models polymerization of multi-functional acrylates, with $k_{p}^{0}$ being a decreasing function of $M$, i.e., the polymerization rate increases with the degree of conversion.

The mass balance equations that govern the above kinetics scheme are

$$
\begin{aligned}
& \frac{\partial I}{\partial t}+k_{d} I=0, \\
& \frac{\partial R}{\partial t}-2 f k_{d} I+k_{p} R M+k_{t} R P^{*}=0, \\
& \frac{\partial M}{\partial t}+k_{p} R M+k_{p} M P^{*}=0, \\
& \frac{\partial P^{*}}{\partial t}-k_{p} R M+k_{t} R P^{*}+k_{t} P^{* 2}=0, \\
& \frac{\partial P}{\partial t}-k_{t} R P^{*}-k_{t} P^{* 2}=0 .
\end{aligned}
$$

Here $I, R, M, P$ are the concentrations in $\mathrm{mol} / \mathrm{L}$ of the reacting species, $t$ is the time, and $P^{*}$ is the total concentration of polymer radicals $\widetilde{P}_{n}^{*}, n=1,2, \ldots$ (We do not distinguish between polymer radicals of different lengths). We remark that since Equation (1e) decouples from the remaining equations we restrict ourselves to studying kinetic Equations (1a-1d).

The rate of production and consumption of radicals (both initiator and polymer radicals) is more significant than the overall rate of change of the radical concentration, which allows us to reduce the differential equations to algebraic balances. This is known as the steady-state assumption (it has been justified in the context of a frontal polymerization problem in [8]), and it reduces the equations $(1 \mathrm{~b}-1 \mathrm{~d})$ to

$$
\frac{\partial M}{\partial t}+k_{e} \sqrt{I} M=0
$$

where

$$
k_{e}=k_{e}^{0} \exp \left\{-E_{e} /\left(R_{g} T\right)\right\}
$$

is the effective reaction rate and

$$
k_{e}^{0}=k_{p}^{0} \sqrt{\frac{2 f k_{d}^{0}}{k_{t}^{0}}}, \quad E_{e}=E_{p}+\frac{E_{d}-E_{t}}{2} .
$$

Note that $k_{e}^{0}$ is a function of $M$. Since $k_{p}^{0}$ is a decreasing function of $M$, while $k_{t}^{0}$ is an increasing function, the effective frequency factor $k_{e}^{0}$ is a decreasing function of $M$. Thus, $k_{e}$ can be written in the form

$$
k_{e}=\Phi(M) k_{\mathrm{eff}}(T), \quad k_{\mathrm{eff}}(T)=k_{\mathrm{eff}}^{0} \exp \left\{-E_{e} /\left(R_{g} T\right)\right\} .
$$

Next, we need to formulate the energy balance in the system. Since the main heat producing step is the propagation step [9], the heat equation has the form

$$
\frac{\partial T}{\partial t}=\kappa \nabla^{2} T-q \frac{\partial M}{\partial t} .
$$

Here $\kappa$ is the thermal diffusivity, $q=-\Delta H /(c \rho)$ is the rise in temperature induced per unit concentration of reacted monomer, $\Delta H$ the enthalpy of the propagation reaction, $c$ the 
specific heat and $\rho$ the mixture density. Thus, the system of equations to be studied is given by (1a), (2), (3). We remark that mass diffusion of the species is neglected compared to heat diffusion because mass-diffusion coefficients in liquids are typically two orders of magnitude smaller than thermal diffusivities.

The spatial region that we consider is a cylindrical shell of circumference $L$, in which the polymerization wave propagates longitudinally. A two-dimensional shell is used rather than a more realistic three-dimensional circular cylinder, because the 2D problem is simpler than its 3D counterpart, yet it still exhibits many of the same properties as the 3D problem. In a fixed coordinate frame $(\tilde{x}, y),-\infty<\tilde{x}<\infty, 0<y<L$, the front propagates along the $\tilde{x}$-axis in the direction of decreasing $\tilde{x}$. By introducing a moving coordinate system $x=\tilde{x}-\varphi(y, t)$ where $\varphi$ is the location of the reaction front at time $t$, we fix the front at $x=0$. The dependent variables in our model are the temperature $T(x, y, t)$, monomer concentration $M(x, y, t)$, initiator concentration $I(x, y, t)$ and velocity of the propagating front $\varphi_{t} \equiv \partial \varphi(y, t) / \partial t<0$. We rewrite the equations in the moving coordinate system to obtain

$$
\begin{aligned}
& \frac{\partial I}{\partial t}-\varphi_{t} \frac{\partial I}{\partial x}+k_{d}(T) I=0 \\
& \frac{\partial M}{\partial t}-\varphi_{t} \frac{\partial M}{\partial x}+k_{\mathrm{eff}}(T) \Phi(M) \sqrt{I} M=0, \\
& \frac{\partial T}{\partial t}-\varphi_{t} \frac{\partial T}{\partial x}=\kappa \nabla^{2} T+q k_{\mathrm{eff}}(T) \Phi(M) \sqrt{I} M=0 .
\end{aligned}
$$

Here $\nabla^{2}$ is the Laplacian in the moving coordinate system defined as

$$
\nabla^{2}=\frac{\partial^{2}}{\partial y^{2}}+\left(1+\varphi_{y}^{2}\right) \frac{\partial^{2}}{\partial x^{2}}-2 \varphi_{y} \frac{\partial^{2}}{\partial x \partial y}-\varphi_{y y} \frac{\partial}{\partial x} .
$$

Boundary conditions far ahead of the front correspond to the initial state of the mixture, i.e.,

$$
x=-\infty: \quad T=T_{0}, \quad M=M_{0}, \quad I=I_{0},
$$

whereas far behind the front

$$
x=+\infty: \quad \frac{\partial T}{\partial x}=0 .
$$

We utilize periodic boundary conditions in $y$ for both $T$ and $\varphi$.

After substituting $J=\sqrt{I}$ in (4), (5) and (6), we obtain

$$
\begin{aligned}
& \frac{\partial J}{\partial t}-\varphi_{t} \frac{\partial J}{\partial x}+J k_{1}(T)=0, \\
& \frac{\partial M}{\partial t}-\varphi_{t} \frac{\partial M}{\partial x}+\Phi(M) J M H\left(J_{0}-J\right) k_{2}(T)=0, \\
& \frac{\partial T}{\partial t}=\kappa \nabla^{2} T+\varphi_{t} \frac{\partial T}{\partial x}+q \Phi(M) J M H\left(J_{0}-J\right) k_{2}(T) .
\end{aligned}
$$

Here

$$
\begin{aligned}
& k_{1}(T)=k_{d}(T) / 2=k_{01} \mathrm{e}^{-E_{1} /\left(R_{g} T\right)}, \quad k_{01}=k_{d}^{0} / 2, \quad E_{1}=E_{d}, \\
& k_{2}(T)=k_{\mathrm{eff}}(T)=k_{02} \mathrm{e}^{-E_{2} /\left(R_{g} T\right)}, \quad k_{02}=k_{\mathrm{eff}}^{0}, \quad E_{2}=E_{e}
\end{aligned}
$$


and $H\left(J_{0}-J\right)$ is the step function

$$
H(\xi)= \begin{cases}0, & \xi \leq 0 \\ 1, & \xi>0\end{cases}
$$

introduced to prevent the inaccuracy of the steady-state approximation which allows the polymerization reaction to occur before initiator is decomposed, i.e., at $J=J_{0}$.

Another simplification in frontal polymerization studies comes from combustion theory. In combustion theory, non-dimensional parameters, such as $R_{g} T_{b} / E_{d}$, determine the structure of the wave. Exploiting the fact that these parameters are small, one can replace the temperature-dependent, Arrhenius reaction rate constants with a simpler function where the integral of this new function over the temperature domain is approximately the same as the original function and the maximum heights of the two functions are equal. This approach is somewhat different from a formal asymptotic analysis in the limit of large activation energy, which is traditional in the combustion theory [10]. The essence of the approach lies in the understanding that an appropriately non-dimensionalized Arrhenius exponential can be treated as an approximation to the 'one-sided' delta-function of the temperature. Consequently, it can be replaced by another function, which is also an approximation to the delta-function. This approach is particularly useful in the case of multiple reaction schemes, i.e., when more than one Arrhenius exponential occurs in the problem, since standard matched asymptotic expansions rarely produce results uniformly valid with respect to multiple small parameters. Employing this technique leads to approximate analytic solutions known to be very accurate. Though frontal polymerization is much slower and significantly less exothermic than combustion processes, parameters of this form also determine the wave structure for frontal polymerization and happen to be small. Therefore, this technique can been used to determine approximate solutions for frontal polymerization. In fact, it has been used to study FP problems yielding accurate results $[8,11-14]$.

The simpler function $K_{i}(T)$ with which we replace the Arrhenius dependence of reaction rate on temperature $k_{i}(T)$ is

$$
K_{i}(T)=\left\{\begin{array}{c}
0, T<T_{i}^{*} \\
A_{i}, T>T_{i}^{*}
\end{array},\right.
$$

where

$$
A_{i}=k_{i}\left(T_{b}\right)=k_{0 i} \exp \left\{-E_{i} /\left(R_{g} T_{b}\right)\right\}
$$

is the Arrhenius function for decomposition $(i=1)$ and polymerization $(i=2)$ reactions, evaluated at $T=T_{b}$, with $k_{0 i}$ and $E_{i}$ being the frequency factors and activation energies of both processes. Here $T_{b}$ is the characteristic temperature at the reaction zone. Since the reaction zone is thin, the temperature does not significantly vary inside the reaction zone, and $T_{b}$, which must be determined in the course of solution of the problem, can be referred to as the reaction zone temperature. In the moving coordinate system $T_{b}$ is the temperature at $x=0$. Next, $T_{i}^{*}=T_{b}\left(1-\epsilon_{i}\right)$ is the temperature at which the first and second reactions begin, and $\epsilon_{i}=R_{g} T_{b} / E_{i}$ is a small dimensionless parameter related to the activation energy. We note that the height of the chosen step function is equal to the maximum of the Arrhenius temperaturedependent function. We have chosen the temperature $T_{i}^{*}$ in such a way that the step function $K_{i}(T)$ and the Arrhenius function $k_{i}(T)$ have approximately equal integral values over the interval $T_{0}<T<T_{b}$. In particular, integrating the function in (13) gives

$$
\int_{T_{0}}^{T_{b}} K_{i}(T) \mathrm{d} T=\frac{R_{g} T_{b}^{2}}{E_{i}} k_{i}\left(T_{b}\right)
$$


and it can be shown by Laplace's method that

$$
\int_{T_{0}}^{T_{b}} k_{i}(T) \mathrm{d} T \approx \frac{R_{g} T_{b}^{2}}{E_{i}} k_{i}\left(T_{b}\right) .
$$

We further simplify the problem making use of the fact that the activation energies of the decomposition and polymerization reactions are large, which results in narrow reaction zones. In the limit of infinite activation energy the reaction zone shrinks to a moving surface, termed a front. In this case Equations (10-12) must be solved without the reaction term both ahead of and behind the reaction front and matched at the reaction front by satisfying matching conditions. Thus, we solve the reactionless equations

$$
\begin{aligned}
& \frac{\partial T}{\partial t}-\varphi_{t} \frac{\partial T}{\partial x}=\kappa \nabla^{2} T, \\
& \frac{\partial M}{\partial t}-\varphi_{t} \frac{\partial M}{\partial x}=0, \\
& \frac{\partial J}{\partial t}-\varphi_{t} \frac{\partial J}{\partial x}=0,
\end{aligned}
$$

both ahead of $(x<0,0<y<L)$ and behind $(x>0,0<y<L)$ the front. Boundary conditions far ahead of the front are

$$
x=-\infty: \quad T=T_{0}, \quad M=M_{0}, \quad J=J_{0}
$$

whereas far behind the front

$$
x=+\infty: \quad T_{x}=0 .
$$

We assume periodic boundary conditions in $y$ for both $T$ and $\varphi$. The matching conditions that are derived in Appendix A have the form

$$
[T]=0, \quad \kappa\left[T_{x}\right]=\frac{q\left(M_{0}-M_{b}\right) \varphi_{t}}{1+\varphi_{y}^{2}}, \quad \frac{\varphi_{t}^{2}}{1+\varphi_{y}^{2}}=F\left(T_{b}\right), \quad M_{b}=f\left(T_{b}\right) .
$$

The brackets denote a jump in a quantity across the front

$$
[v]=v\left(x=0^{+}\right)-v\left(x=0^{-}\right),
$$

$T_{b}$ and $M_{b}$ are the temperature and monomer concentration at the front, respectively, and the functions $f$ and $F$ are given by

$$
\begin{aligned}
& F\left(T_{b}\right) \equiv \frac{\kappa k_{01} R_{g} T_{b}^{2}\left(1-\alpha M_{0}+\alpha M_{0} \exp \left(-j_{0}\right)\right)}{q M_{0} E_{1}} \\
& \quad \times \exp \left(j_{0}-\frac{E_{1}}{R_{g} T_{b}}\right)\left(\int_{0}^{j_{0}}\left(\frac{\mathrm{e}^{\eta}-1}{1+\left(\frac{\alpha M_{0}}{1-\alpha M_{0}}\right) \mathrm{e}^{\eta-j_{0}}}\right) \frac{1}{\eta} \mathrm{d} \eta\right)^{-1} \\
& f\left(T_{b}\right)= M_{0} \exp \left(-j_{0}\right)\left(\frac{1}{1-\alpha M_{0}+\alpha M_{0} \exp \left(-j_{0}\right)}\right), \\
& j_{0}=\frac{J_{0} A_{2}}{A_{1}}, \quad A_{1}=k_{1}\left(T_{b}\right), \quad A_{2}=k_{2}\left(T_{b}\right) .
\end{aligned}
$$


The expressions for $f$ and $F$ have been derived for a specific form of the function $\Phi$, namely, $\Phi(M)=1-\alpha M$, where $\alpha>0$ is a parameter that characterizes the autoacceleration of the reaction. We restrict ourselves to considering this simple linear dependence as we are only interested in the qualitative effect of autoacceleration. One can think of this function as a result of linearization of a more realistic dependence $\Phi$.

We remark that kinetic parameter values typical of the polymerization processes are such that both decomposition and polymerization reactions occur in the same reaction zone. In general, it does not have to be the case, i.e., different reactions can occur at different spatial locations as known from the combustion literature [15-17]. Below we determine stationary solutions of the above problem, which correspond to uniformly propagating one-dimensional traveling waves in the original problem, and then perform their linear and weakly nonlinear stability analyses.

\section{Basic solution and its stability}

The stationary solution in $1 \mathrm{D}$ is

$$
\begin{aligned}
& \widehat{T}(x)= \begin{cases}T_{0}+\left(\widehat{T}_{b}-T_{0}\right) \exp (\widehat{u} x / \kappa), & x<0 \\
\widehat{T}_{b}, & x>0\end{cases} \\
& \widehat{M}(x)=\left\{\begin{array}{ll}
M_{0}, & x<0 \\
\widehat{M}_{b}, & x>0
\end{array}, \quad \widehat{J}(x)=\left\{\begin{array}{ll}
J_{0}, & x<0 \\
0, & x>0
\end{array}, \quad \widehat{\varphi}_{t}=-\widehat{u}>0,\right.\right.
\end{aligned}
$$

where

$$
\widehat{T}_{b}=T_{0}+q\left(M_{0}-\widehat{M}_{b}\right), \quad \widehat{M}_{b}=f\left(\widehat{T}_{b}\right), \quad \widehat{u}^{2}=F\left(\widehat{T}_{b}\right) .
$$

To determine stability of the stationary solution we substitute

$$
\begin{aligned}
& T=\widehat{T}+\delta \widetilde{T}=\widehat{T}(x)+\delta \exp (\omega t+\mathrm{i} k y) \theta(x), \\
& M=\widehat{M}+\delta \widetilde{M}=\widehat{M}(x)+\delta \exp (\omega t+\mathrm{i} k y) m(x), \\
& \varphi=-\widehat{u} t+\delta \widetilde{\varphi}=-\widehat{u} t+\delta \exp (\omega t+\mathrm{i} k y)
\end{aligned}
$$

in the equations and matching conditions and linearize in small $\delta$. Here $k=2 \pi j / L, j=$ $1,2, \ldots$ is the wavenumber and $\omega$ is the temporal frequency of perturbations, and $\delta$ is a small magnitude of perturbations.

This results in the dispersion relation

$$
4 \Omega^{3}+\left(1+4 s^{2}+4 z_{1}-\left(z_{1}-P_{1}\right)^{2}\right) \Omega^{2}+z_{1}\left(1+4 s^{2}+P_{1}\right) \Omega+s^{2} z_{1}^{2}=0 .
$$

Here $s=\kappa k / \widehat{u}$ is the non-dimensional wavenumber and $\Omega=\kappa \omega / \widehat{u}^{2}$ is the non-dimensional frequency of oscillation. A detailed account of the linear stability analysis can be found in $[3,5,6]$. We use the following non-dimensional parameters in the analysis

$$
\begin{aligned}
& z_{1}=\frac{F^{\prime}\left(\widehat{T}_{b}\right)\left(\widehat{T}_{b}-T_{0}\right)}{F\left(\widehat{T}_{b}\right)} \equiv 2\left(\widehat{T}_{b}-T_{0}\right) \frac{\partial \log \widehat{u}}{\partial \widehat{T}_{b}}, \quad z_{2}=\frac{F^{\prime \prime}\left(\widehat{T}_{b}\right)\left(\widehat{T}_{b}-T_{0}\right)^{2}}{2 F\left(\widehat{T}_{b}\right)}, \\
& z_{3}=\frac{F^{\prime \prime \prime}\left(\widehat{T}_{b}\right)\left(\widehat{T}_{b}-T_{0}\right)^{3}}{6 F\left(\widehat{T}_{b}\right)}, \quad P_{1}=q f^{\prime}\left(\widehat{T}_{b}\right) \equiv q \frac{\partial \widehat{M}_{b}}{\partial \widehat{T}_{b}}, \\
& P_{2}=\frac{1}{2} q\left(\widehat{T}_{b}-T_{0}\right) f^{\prime \prime}\left(\widehat{T}_{b}\right), \quad P_{3}=\frac{1}{6} q\left(\widehat{T}_{b}-T_{0}\right)^{2} f^{\prime \prime \prime}\left(\widehat{T}_{b}\right) .
\end{aligned}
$$






Figure 1. Neutral stability curves $z_{1 c r}(s)$ in the $\left(s, z_{1}\right)$ plane for various values of $P_{1}$. Above respective curves, uniform propagation is unstable.

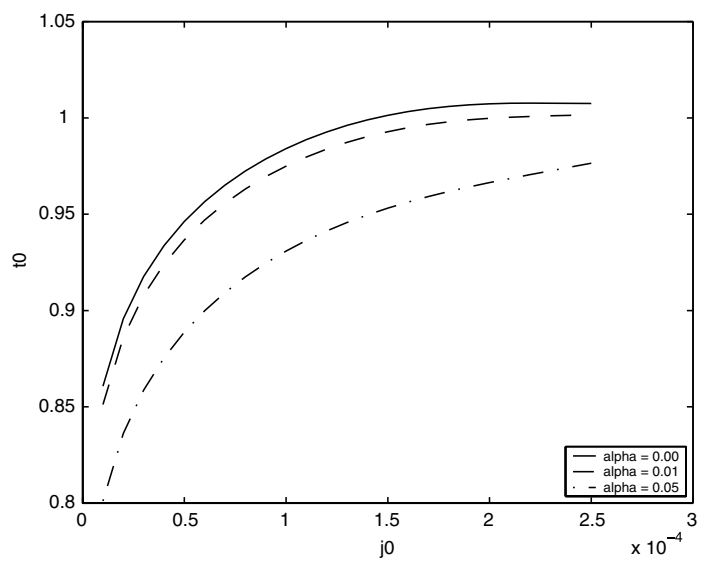

Figure 2. Stability curves in the $\left(j_{0}, t_{0}\right)$ plane for $s=0.55$ and $\alpha=0.00,0.01,0.05$ in descending order. Parameter values are given in (18).

Instability occurs when a pair of complex conjugate eigenvalues crosses the imaginary axis as the parameters vary. At the stability boundary

$$
\begin{aligned}
z_{1 c r}= & 4+2 P_{1}-4 s^{2}\left(1+4 s^{2}+P_{1}\right)^{-1} \\
& +2\left(\left(2+P_{1}-2 s^{2}\left(1+4 s^{2}+P_{1}\right)^{-1}\right)^{2}-P_{1}^{2}+1+4 s^{2}\right)^{\frac{1}{2}}, \\
\omega_{0}^{2}= & (\Im \mathfrak{m} \Omega)^{2}=\frac{1}{8} z_{1 c r}\left(1+4 s^{2}+P_{1}\right) .
\end{aligned}
$$

The neutral stability curve in the $\left(s, z_{1}\right)$ plane has a minimum at $s=s_{m}>0$ for all $P_{1} \geq 0$. Neutral stability curves are shown in Figure 1 for physically meaningful values of $P_{1}$. Note that the polymerization wave becomes more stable as $P_{1}$ increases.

To investigate the influence of the autoacceleration on linear stability of a model system, we set

$$
\left(E_{1}-E_{2}\right) /\left(R_{g} q M_{0}\right)=19.70, \quad E_{1} /\left(R_{g} q M_{0}\right)=52.95 .
$$

These are typical values for MMA polymerization with 2,2'-azo-bis-isobutyronitrile (AIBN) as an initiator [18]. Figure 2 shows the stability curves in the $\left(2_{0}, j_{0}\right)$ plane for $s=0.55$ and for various $\alpha$-values. Here $j_{0}$ is given by (14) and $t_{0}=T_{0} /\left(q M_{0}\right)$ is the nondimensional initial temperature. An increase in the value of $\alpha$ corresponds to increased autoacceleration. The linearly unstable region corresponds to the area under the graphs. As depicted in Figure 2, autoacceleration was found to promote linear stability of the system. This is not surprising because instability usually occurs with slowly propagating waves, near extinction limits, while autoacceleration enhances propagation. Similar results can be seen in Figure 3, which corresponds to

$$
\left(E_{1}-E_{2}\right) /\left(R_{g} q M_{0}\right)=22.65, \quad E_{1} /\left(R_{g} q M_{0}\right)=58.86,
$$

typical values for the initiator tert-butyl peroxide (TBPO) [8].

\section{Weakly nonlinear analysis}

We perform a nonlinear analysis that will allow us to obtain amplitude equations to characterize the evolution of the unstable modes. Gross and Volpert [5] have studied the 1D case 


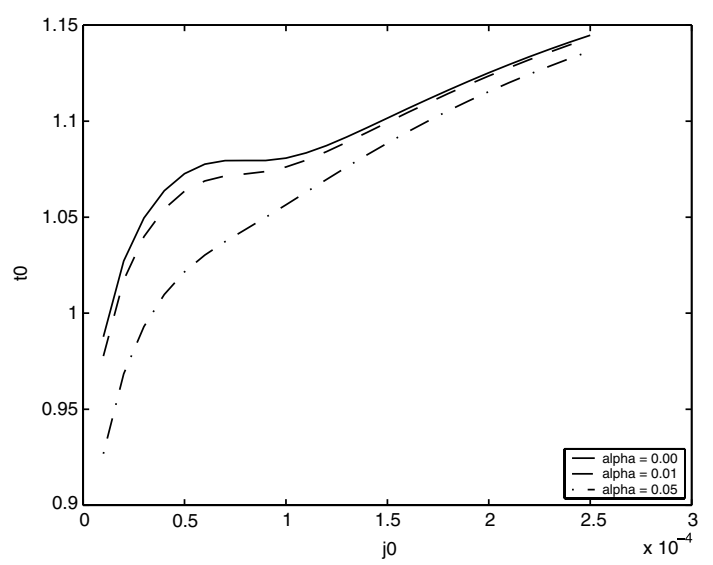

Figure 3. Stability curves in the $\left(j_{0}, t_{0}\right)$ plane for $s=0.55$ and $\alpha=0.00,0.01,0.05$ in descending order. Parameter values are given in (19).

for loss of neutral stability at the wavenumber $s=0$. This corresponds to small values of the tube circumference $L$. The analysis in this instance results in a single Landau-Stuart equation which governs the weakly unstable modes. If, however, the tube circumference $L$ is larger, loss of stability will occur for some $s>0$. We perform a weakly nonlinear stability analysis in a neighborhood of the minimum of the neutral stability curve, i.e., for a fixed value of $P_{1}$ we take $s$ close to $s_{m}$ and $z_{1}$ close to $z_{1 c r}(s)$. The form of the bifurcating solution, e.g. the number $j$ of maxima of the temperature in the $\eta$-direction, which is determined by the wavenumber $k$, will therefore depend on the value of the shell circumference $L$ according to the equation

$$
j=\frac{\hat{u} s}{\kappa} \frac{L}{2 \pi} .
$$

Our nonlinear analysis yields a coupled set of Landau amplitude equations.

We introduce time scales $t_{0}=t, t_{1}=\epsilon t, t_{2}=\epsilon^{2} t$ and expand $T, M$, and $\varphi$ as

$$
\begin{aligned}
& T=\widehat{T}+\epsilon T_{1}+\epsilon^{2} T_{2}+\epsilon^{3} T_{3}+\cdots, \\
& M=\widehat{M}+\epsilon M_{1}+\epsilon^{2} M_{2}+\epsilon^{3} M_{3}+\cdots, \\
& \varphi=-\widehat{u} t_{0}+\epsilon \varphi_{1}+\epsilon^{2} \varphi_{2}+\epsilon^{3} \varphi_{3}+\cdots
\end{aligned}
$$

Also $z_{1}=z_{1 c r}+\mu \epsilon^{2}$. To non-dimensionalize the above the following scales are used, where $j=0,1,2$

$$
\begin{aligned}
& \xi=\frac{\widehat{u}}{\kappa} x, \quad \eta=\frac{\widehat{u}}{\kappa} y, \quad \psi=\frac{\widehat{u}}{\kappa} \varphi, \quad \psi_{j}=\frac{\widehat{u}}{\kappa} \varphi_{j}, \quad t_{j}=\frac{\kappa}{\widehat{u}^{2}} \tau_{j}, \quad \theta=\frac{T}{\widehat{T}_{b}-T_{0}}, \\
& \theta_{b}=\frac{T_{b}}{\widehat{T}_{b}-T_{0}}, \quad m=\frac{M}{-\widehat{M}_{b}+M_{0}}, \quad m_{b}=\frac{M_{b}}{-\widehat{M}_{b}+M_{0}}, \quad m_{j b}=\frac{M_{j b}}{-\widehat{M}_{b}+M_{0}} .
\end{aligned}
$$

Consequently, we have the following sequence of problems

$$
\begin{aligned}
& \frac{\partial \theta_{j}}{\partial \tau_{0}}+\frac{\partial \theta_{j}}{\partial \xi}-\left(\frac{\partial^{2} \theta_{j}}{\partial \xi^{2}}+\frac{\partial^{2} \theta_{j}}{\partial \eta^{2}}\right)-\left(\frac{\partial \psi_{j}}{\partial \tau_{0}}+\frac{\partial^{2} \psi_{j}}{\partial \eta^{2}}\right) \frac{\mathrm{d} \widehat{\theta}}{\mathrm{d} \xi}=\widetilde{Q}_{j}, \\
& {\left[\theta_{j}\right]=0, \quad\left[\frac{\partial \theta_{j}}{\partial \xi}\right]-\frac{\partial \psi_{j}}{\partial \tau_{0}}-m_{j b}=\widetilde{R}_{j},}
\end{aligned}
$$




$$
2 \frac{\partial \psi_{j}}{\partial \tau_{0}}+z_{1 c r} \theta_{j b}=\widetilde{S}_{j}, \quad m_{j b}-P_{1} \theta_{j b}=\widetilde{T}_{j}
$$

where $\widetilde{Q}_{j}, \widetilde{R}_{j}, \widetilde{S}_{j}$ and $\widetilde{T}_{j}$ are given in the Appendix B. The solution $\theta_{j}, \psi_{j}$ satisfy periodic boundary conditions in $\eta$ and

$$
\left.\frac{\partial \theta_{j}}{\partial \xi}\right|_{\xi=+\infty}=0,\left.\quad \theta_{j}\right|_{\xi=-\infty}=0
$$

The solvability condition for problem $(20-23)$ is

$$
\begin{aligned}
& \int_{0}^{\frac{2 \pi}{\omega_{0}}} \int_{0}^{L}\left(\left.\bar{v}\right|_{\xi=0}\left(\frac{P_{1}}{z_{1 c r}} \widetilde{S}_{j}+\widetilde{R}_{j}+\widetilde{T}_{j}\right)-\frac{1}{z_{1 c r}} \widetilde{S}_{j}\left[\frac{\partial \bar{v}}{\partial \xi}\right]\right) \mathrm{d} \eta \mathrm{d} \tau_{0} \\
& \quad=\int_{0}^{\frac{2 \pi}{\omega_{0}}} \int_{0}^{L} \int_{-\infty}^{\infty} \widetilde{Q}_{j} \bar{v} \mathrm{~d} \tau_{0} \mathrm{~d} \eta \mathrm{d} \xi
\end{aligned}
$$

where $v$ is a solution of the adjoint problem given by

$$
\begin{aligned}
& v_{ \pm}\left(\xi, \eta, \tau_{0}\right)=\left\{\begin{array}{ll}
\exp \left(\mathrm{i} \omega_{0} \tau_{0} \pm \mathrm{i} s \eta+\frac{1}{2}(-1+\bar{d}) \xi\right), & \xi<0 \\
\exp \left(\mathrm{i} \omega_{0} \tau_{0} \pm \mathrm{i} s \eta+\frac{1}{2}(-1-\bar{d}) \xi\right), & \xi>0
\end{array},\right. \\
& v_{0}(\xi)=\left\{\begin{array}{cc}
1, & \xi<0 \\
\exp (-\xi), & \xi>0
\end{array}\right.
\end{aligned}
$$

and

$$
d=\sqrt{1+4 \Omega+4 s^{2}}
$$

4.1. The $O(\epsilon)$ Problem $(j=1)$

The solution of the $O(\epsilon)$ problem is

$$
\begin{aligned}
& \theta_{1}=\left(A \mathrm{e}^{\mathrm{i}\left(\omega_{0} \tau_{0}+s \eta\right)}+B \mathrm{e}^{\mathrm{i}\left(\omega_{0} \tau_{0}-s \eta\right)}\right) X_{1}(\xi)+C C, \\
& \psi_{1}=\frac{A z_{1} c r}{2} \mathrm{e}^{\mathrm{i}\left(\omega_{0} \tau_{0}+s \eta\right)}+\frac{B z_{1} r r}{2} \mathrm{e}^{\mathrm{i}\left(\omega_{0} \tau_{0}-s \eta\right)}+C C+\mho,
\end{aligned}
$$

where $\mathrm{CC}$ denotes the complex conjugate, $\mho, A$ and $B$ are functions of the slow times and

$$
X_{1}(\xi)=\left\{\begin{array}{ll}
-\left(\mathrm{i} \omega_{0}+\frac{1}{2} z_{1 c r}\right) \mathrm{e}^{\frac{1}{2}(1+d) \xi}+\frac{1}{2} z_{1 c r} \mathrm{e}^{\xi}, & \xi<0 \\
-\mathrm{i} \omega_{0} \mathrm{e}^{\frac{1}{2}(1-d) \xi}, & \xi>0
\end{array} .\right.
$$

4.2. The $O\left(\epsilon^{2}\right)$ PRoblem $(j=2)$

Applying the solvability condition to the $O\left(\epsilon^{2}\right)$ problem with $v=v_{+}$and $v=v_{-}$shows that $A$ and $B$ depend only on the slow time $\tau_{2}$. When $v=v_{0}$ the solvability condition yields

$$
\partial \mho / \partial \tau_{1}=r_{0}\left(|A|^{2}+|B|^{2}\right)
$$


with $r_{0}$ as

$$
\begin{aligned}
r_{0}=\frac{z_{1 c r}}{-2\left(P_{1}+1\right)}\{ & -2 \omega_{0}^{2}\left(\left(\frac{P_{1}+1}{z_{1 c r}}\right)\left(\frac{1}{4} z_{1 c r}^{2}-z_{2}\right)+\frac{1}{2} z_{1 c r} P_{1}+P_{2}\right) \\
& -\frac{s^{2} z_{1 c r}^{2}}{2}\left(1-\frac{P_{1}+1}{z_{1 c r}}\right)-\frac{z_{1 c r} \omega_{0}^{2}}{2}\left(2+\frac{1-\bar{d}}{1+\bar{d}}+\frac{1-d}{1+d}\right) \\
& \left.-\frac{s^{2} z_{1 c r} \mathrm{i} \omega_{0}}{2}\left(\frac{1-\bar{d}}{1+\bar{d}}-\frac{1-d}{1+d}\right)+\frac{s^{2}}{2} z_{1 c r}^{2}\right\} .
\end{aligned}
$$

The solution for the $O\left(\epsilon^{2}\right)$ problem is

$$
\begin{aligned}
\theta_{2}= & g_{0}(\xi)\left(|A|^{2}+|B|^{2}\right)+\left\{g_{1}(\xi)\left(A^{2} \exp \left(2 \mathrm{i}\left(\omega_{0} \tau_{0}+s \eta\right)\right)+B^{2} \exp \left(2 \mathrm{i}\left(\omega_{0} \tau_{0}-s \eta\right)\right)\right)\right. \\
& \left.+g_{2}(\xi) A B \exp \left(2 \mathrm{i} \omega_{0} \tau_{0}\right)+g_{3}(\xi) A \bar{B} \exp (2 \mathrm{i} s \eta)+C C\right\} \\
\psi_{2}= & C_{0}\left(|A|^{2}+|B|^{2}\right)+\left\{C_{1}\left(A^{2} \exp \left(2 \mathrm{i}\left(\omega_{0} \tau_{0}+s \eta\right)\right)+B^{2} \exp \left(2 \mathrm{i}\left(\omega_{0} \tau_{0}-s \eta\right)\right)\right)\right. \\
& \left.+C_{2} A B \exp \left(2 \mathrm{i} \omega_{0} \tau_{0}\right)+C_{3} A \bar{B} \exp (2 \mathrm{i} s \eta)+C C\right\},
\end{aligned}
$$

where the functions $g_{j}(\xi)$ are

$$
\begin{aligned}
& g_{0}= \begin{cases}D_{01} \exp (\xi)+D_{02}(\exp (\xi)+\xi \exp (\xi))+\left(D_{03} \exp \left((1+d) \frac{\xi}{2}\right)+C C\right), & \xi<0 \\
a_{02}+\left(D_{04} \exp \left((1-d) \frac{\xi}{2}\right)+C C\right), & \xi>0\end{cases} \\
& g_{1}= \begin{cases}a_{11} \exp \left(\left(1+d_{1}\right) \frac{\xi}{2}\right)+D_{11} \exp (\xi)+D_{13} \exp \left((1+d) \frac{\xi}{2}\right), & \xi<0, \\
a_{12} \exp \left(\left(1-d_{1}\right) \frac{\xi}{2}\right)+D_{14} \exp \left((1-d) \frac{\xi}{2}\right), & \xi>0\end{cases} \\
& g_{2}= \begin{cases}a_{21} \exp \left(\left(1+d_{2}\right) \frac{\xi}{2}\right)+D_{21} \exp (\xi)+D_{23} \exp \left((1+d) \frac{\xi}{2}\right), & \xi<0, \\
a_{22} \exp \left(\left(1-d_{2}\right) \frac{\xi}{2}\right)+D_{24} \exp \left((1-d) \frac{\xi}{2}\right), & \xi>0\end{cases} \\
& g_{3}= \begin{cases}a_{31} \exp \left(\left(1+d_{3}\right) \frac{\xi}{2}\right)+D_{31} \exp (\xi)+\left(D_{33} \exp \left((1+d) \frac{\xi}{2}\right)+C C\right), & \xi<0, \\
a_{32} \exp \left(\left(1-d_{3}\right) \frac{\xi}{2}\right)+\left(D_{34} \exp \left((1-d) \frac{\xi}{2}\right)+C C\right), & \xi>0\end{cases}
\end{aligned}
$$

Here

$$
d_{1}=\sqrt{1+8 \mathrm{i} \omega_{0}+16 s^{2}}, \quad d_{2}=\sqrt{1+8 \mathrm{i} \omega_{0}}, \quad d_{3}=\sqrt{1+16 s^{2}}
$$

and the coefficients $a_{i j}, D_{i j}$ and $C_{j}$ are given in Appendix B.

4.3. The $O\left(\epsilon^{3}\right)$ Problem $(j=3)$

The solvability conditions for the $O\left(\epsilon^{3}\right)$ problem yield a coupled set of Landau equations

$$
\frac{\partial A}{\partial \tau_{2}}=\mu A \chi+A^{2} \bar{A} \beta_{1}+A B \bar{B} \beta_{2}, \quad \frac{\partial B}{\partial \tau_{2}}=\mu B \chi+B^{2} \bar{B} \beta_{1}+A B \bar{A} \beta_{2} .
$$

The complex coefficients $\beta_{1}, \beta_{2}$ and $\chi$ are given in the Appendix B. Amplitude equations of this form have been derived in many problems, e.g. in the context of combustion problems $[19,20]$. 


\section{Analysis of the amplitude equations}

We let the amplitudes $A$ and $B$ of $\psi_{1}$, which determines the shape of the front, be of the form

$$
A\left(\tau_{2}\right)=a\left(\tau_{2}\right) \exp \left(\mathrm{i} \theta_{a} \tau_{2}\right), \quad B\left(\tau_{2}\right)=b\left(\tau_{2}\right) \exp \left(\mathrm{i} \theta_{b} \tau_{2}\right) .
$$

Substituting (25) into (24) and separating real and imaginary parts results in

$$
\begin{array}{ll}
\frac{\mathrm{d} a}{\mathrm{~d} \tau_{2}}=\mu \chi_{\mathrm{r}} a+\beta_{1 \mathrm{r}} a^{3}+\beta_{2 \mathrm{r}} a b^{2}, & \frac{\mathrm{d} \theta_{a}}{\mathrm{~d} \tau_{2}}=\mu \chi_{\mathrm{i}}+\beta_{1 \mathrm{i}} a^{2}+\beta_{2 \mathrm{i}} b^{2}, \\
\frac{\mathrm{d} b}{\mathrm{~d} \tau_{2}}=\mu \chi_{\mathrm{r}} b+\beta_{1 \mathrm{r}} b^{3}+\beta_{2 \mathrm{r}} a^{2} b, & \frac{\mathrm{d} \theta_{b}}{\mathrm{~d} \tau_{2}}=\mu \chi_{\mathrm{i}}+\beta_{1 \mathrm{i}} b^{2}+\beta_{2 \mathrm{i}} a^{2} .
\end{array}
$$

Here the subscripts $r$ and $i$ represent the real and imaginary parts of the respective coefficients. In order to determine the steady state solutions of (26), (27) which, in the original problem, correspond to a superposition of waves traveling along the front, we consider $\mathrm{d} a / \mathrm{d} \tau_{2}=\mathrm{d} b / \mathrm{d} \tau_{2}=0$. This leads to

$$
a\left(\mu \chi_{\mathrm{r}}+\beta_{1 \mathrm{r}} a^{2}+\beta_{2 \mathrm{r}} b^{2}\right)=0, \quad b\left(\mu \chi_{\mathrm{r}}+\beta_{1 \mathrm{r}} b^{2}+\beta_{2 \mathrm{r}} a^{2}\right)=0 .
$$

There are four critical points

$$
a_{1}=b_{1}=0, \quad a_{2}=0, \quad b_{2}=w_{t}, \quad a_{3}=w_{t}, \quad b_{3}=0, \quad a_{4}=b_{4}=w_{s},
$$

where

$$
w_{t}=\left(-\mu \chi_{\mathrm{r}} / \beta_{1 \mathrm{r}}\right)^{1 / 2}, \quad w_{s}=\left(-\mu \chi_{\mathrm{r}} /\left(\beta_{1 \mathrm{r}}+\beta_{2 \mathrm{r}}\right)\right)^{1 / 2} .
$$

In the case of the first critical point the amplitudes $A$ and $B$ are identically equal to zero, which corresponds to the uniformly propagating wave in the original problem. The second and third critical points correspond to waves traveling along the front, which are right- and left-traveling waves, respectively. The last critical point corresponds to a standing wave.

It can be shown that for all parameter values $\chi_{\mathrm{r}}>0$. Thus, from the expression for $w_{t}$ we conclude that left- and right-traveling wave exist for $\mu>0$ (the so-called supercritical bifurcation) if $\beta_{1 \mathrm{r}}<0$ and for $\mu<0$ (the subcritical bifurcation) if $\beta_{1 \mathrm{r}}>0$. In a similar way, the supercritical bifurcation of standing waves occurs if $\beta_{1 \mathrm{r}}+\beta_{2 \mathrm{r}}<0$ and subcritical bifurcation occurs if $\beta_{1 \mathrm{r}}+\beta_{2 \mathrm{r}}>0$. All the subcritical bifurcations are known to produce locally unstable regimes. The supercritical bifurcation can lead to either stable or unstable solutions depending on the parameter values. Specifically, the supercritical bifurcation of traveling waves (which occurs if $\left.\beta_{1 \mathrm{r}}<0\right)$ is stable if $\beta_{2 \mathrm{r}}<\beta_{1 \mathrm{r}}$ and unstable otherwise. The supercritical bifurcation of standing waves (which occurs if $\beta_{1 \mathrm{r}}+\beta_{2 \mathrm{r}}<0$ ) is stable if $\beta_{2 \mathrm{r}}>\beta_{1 \mathrm{r}}$ and unstable otherwise.

Comissiong, Gross and Volpert [6] found that both traveling and standing waves result from a supercritical bifurcation for certain initiators. The supercritical traveling wave solution was found to be the stable mode in most parameter regimes, as also observed by experiment [2]. According to the initiator used in the reaction, supercritical standing waves became the stable mode along the front for very specific initiator concentrations. Upon setting $\alpha=0$, we recovered the results of Comissiong, Gross and Volpert [6] for a two-dimensional system without the autoacceleration effect. The quantities $\beta_{1 \mathrm{r}}$ and $\beta_{2 \mathrm{r}}$ were found to be always negative (for all parameter values investigated). We take parameter values (18), typical for the initiator AIBN. With $\alpha=0$, for the most part $\beta_{2 \mathrm{r}}<\beta_{1 \mathrm{r}}<0$, but for a very small range of 


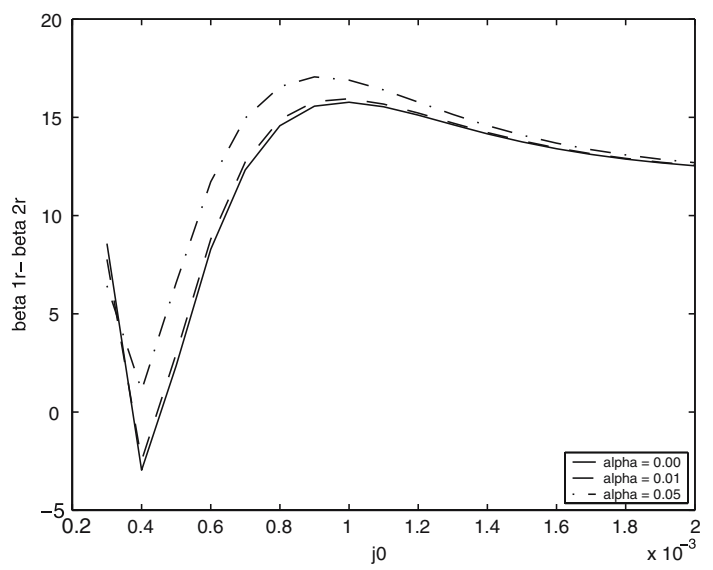

Figure 4. Graphs of $\beta_{1 r}-\beta_{2 r}$ vs. the non-dimensional $j 0=J_{0} \frac{A_{2}}{A_{1}}$ for the initiator AIBN.

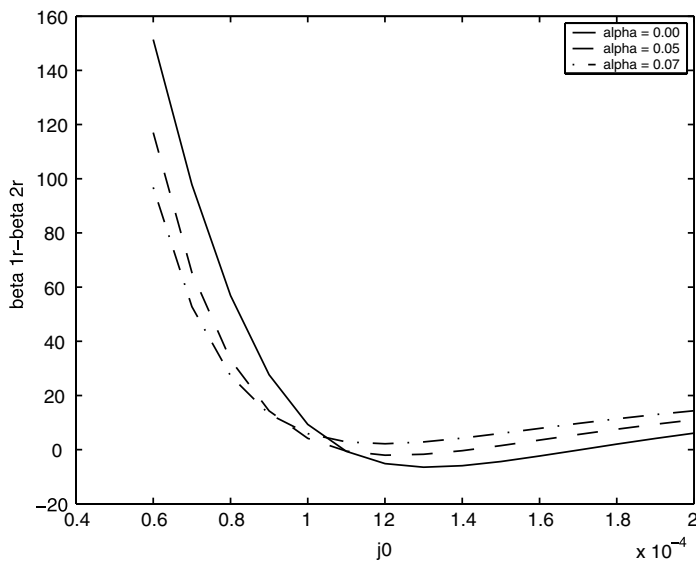

Figure 5. Graphs of $\beta_{1 \mathrm{r}}-\beta_{2 \mathrm{r}}$ vs. the non-dimensional $j 0=J_{0} \frac{A_{2}}{A_{1}}$ for the initiator TBPO.

Table 1. Regimes of existence and stability of equilibrium solutions for AIBN.

\begin{tabular}{llllll}
\hline Initiator & \multirow{2}{*}{$\begin{array}{l}\text { Existence } \\
\text { AIBN }\end{array}$} & Stability & \multicolumn{3}{c}{ Detected } \\
\cline { 4 - 6 } & & & $\alpha=0$ & $\alpha=0.01$ & $\alpha=0.05$ \\
\hline Subcrit. & $\mu<0$ & Unstable & No & No & No \\
TW & $\beta_{1 \mathrm{r}}>0$ & & & \\
Supercrit. & $\mu>0$ & Stable iff & Yes & Yes & Yes \\
TW & $\beta_{1 \mathrm{r}}<0$ & $\beta_{1 \mathrm{r}}>\beta_{2 \mathrm{r}}$ & & & \\
Subcrit. & $\mu<0$ & Unstable & No & No & No \\
SW & $\beta_{1 \mathrm{r}}+\beta_{2 \mathrm{r}}>0$ & & & & \multirow{2}{*}{ No } \\
Supercrit. & $\mu>0$ & Stable iff & Yes & Yes & \\
SW & $\beta_{1 \mathrm{r}}+\beta_{2 \mathrm{r}}<0$ & $\beta_{2 \mathrm{r}}>\beta_{1 \mathrm{r}}$ & & & \\
\hline
\end{tabular}

initiator concentration $j_{0}$ we find that $\beta_{1 \mathrm{r}}<\beta_{2 \mathrm{r}}<0$ (see Table 1 and Figure 4). Supercritical traveling and standing waves are both detected, and for the most part the traveling waves are stable while the standing waves are unstable, except for a very small range where supercritical standing waves become the stable mode of propagation [6].

Upon repeating the investigation for $\alpha \neq 0$, it was found that upon increasing the autoacceleration effect (to $\alpha=0.05$ ), we find that $\beta_{2 \mathrm{r}}<\beta_{1 \mathrm{r}}<0$ always. Hence previously detected stable regimes of supercritical standing waves are eliminated, making the supercritical traveling waves the only stable regime of existence.

We next take parameter values (19), typical for the initiator TBPO [8]. Once more, for $\alpha=0$, we were able to recover the results of Comissiong et al. [6], where supercritical standing waves along the front were the stable mode for a very limited range of initiator concentration. Upon increasing the autoacceleration effect (to $\alpha=0.07$ ), supercritical standing waves were no longer detected (as $\beta_{2 \mathrm{r}}<\beta_{1 \mathrm{r}}<0$ always), making supercritical traveling waves the only stable regime of existence once more (see Table 2 and Figure 5). This agrees with the experimental data in [2] where only traveling waves along the front have been observed.

We remark that the symmetry of the amplitude equations, i.e., the same coefficients in front of similar terms of both equations, is due to symmetries in the original problem, 
Table 2. Regimes of existence and stability of equilibrium solutions for TBPO.

\begin{tabular}{llllll}
\hline Initiator & \multirow{2}{*}{$\begin{array}{l}\text { Existence } \\
\text { TBPO }\end{array}$} & Stability & \multicolumn{3}{c}{ Detected } \\
\cline { 4 - 6 } & & & $\alpha=0$ & $\alpha=0 \cdot 05$ & $\alpha=0.07$ \\
\hline Subcrit. & $\mu<0$ & Unstable & No & No & No \\
TW & $\beta_{1 \mathrm{r}>0}$ & & & & \\
Supercrit. & $\mu>0$ & Stable iff & Yes & Yes & Yes \\
TW & $\beta_{1 \mathrm{r}}<0$ & $\beta_{1 \mathrm{r}}>\beta_{2 \mathrm{r}}$ & & & \\
Subcrit. & $\mu<0$ & Unstable & No & No & No \\
SW & $\beta_{1 \mathrm{r}}+\beta_{2 \mathrm{r}}>0$ & & & & No \\
Supercrit. & $\mu>0$ & Stable iff & Yes & Yes & \\
SW & $\beta_{1 \mathrm{r}}+\beta_{2 \mathrm{r}}<0$ & $\beta_{2 \mathrm{r}}>\beta_{1 \mathrm{r}}$ & & & \\
\hline
\end{tabular}

specifically, due to the translational and reflectional invariance of the governing equations in the $y$-direction. In reality these symmetries may be broken, which will change the behavior of the system in the immediate vicinity of the bifurcation point, but will not qualitatively affect the regimes of propagation slightly away from the bifurcation point.

\section{Conclusion}

We have studied various regimes of propagation of a free-radical polymerization front that bifurcate from a uniformly propagating wave when it loses stability as parameters of the system are varied. We utilize a kinetic scheme which involves the decomposition of initiator molecules to form free radicals, their reactions with monomer units for the formation of polymer chains, and subsequent termination of the growing chains. In addition, we account for autoacceleration of the polymerization process which can occur either due to the gel effect or branching in multi-functional monomers. As autoacceleration is known to enhance polymerization, we model it by appropriately modifying reaction rate constants. Using a variant of large-activation-energy asymptotics, we reduce the problem to a free-boundary problem. We first obtain the basic solutions in the form of uniformly propagating one-dimensional traveling waves. Then we investigate the effect of autoacceleration on the linear and weakly nonlinear stability of the basic state.

We find that the autoacceleration enhances the linear stability of the propagating front, as expected physically. Our weakly nonlinear analysis results in a pair of Landau equations (24), which describes the evolution of the amplitudes of the linearly unstable modes. For the parameters investigated, it has been found that both traveling and standing waves result from a supercritical bifurcation. The traveling-wave solution is found to be the stable mode in most parameter regimes, as observed by experiment. If the autoacceleration effect is absent or mild, then for some types and amounts of the initiator used in the reaction, stable supercritical standing waves can occur. With sufficiently large autoacceleration supercritical standing waves are unstable.

\section{Acknowledgments}

This research has been supported in part by NSF Grants DMS-0103856 and CTS-0138712. Donna Comissiong has been supported by a Fulbright fellowship administered by LASPAU. 


\section{Appendix A: Matching conditions}

In order to derive the matching conditions we study the equations in the reaction zone. The dominant balance in the reaction zone equations is that of the reaction term and the highest spatial derivative (which could be formally derived by stretching the spatial scale in the direction of propagation $x$ )

$$
\begin{aligned}
& -\varphi_{t} \frac{\partial J}{\partial x}+J A_{1}=0, \\
& -\varphi_{t} \frac{\partial M}{\partial x}+J M(1-\alpha M) A_{2}=0, \\
& \kappa\left(1+\varphi_{y}^{2}\right) \frac{\partial^{2} T}{\partial x^{2}}+q J M(1-\alpha M) A_{2}=0 .
\end{aligned}
$$

Combining Equations (A.2) and (A.3) results in

$$
\kappa\left(1+\varphi_{y}^{2}\right) \frac{\partial^{2} T}{\partial x^{2}}-q u \frac{\partial M}{\partial x}=0 .
$$

Integrating (A.4) from a point $x$ in the reaction zone to the product end of it, where $M=M_{b}$ (the final concentration of the monomer), and $T_{x}$ is essentially zero gives

$$
\kappa \frac{\partial T}{\partial x}=-\frac{q\left(M-M_{b}\right) \varphi_{t}}{1+\varphi_{y}^{2}} .
$$

Furthermore, integrating (A.4) across the reaction zone gives

$$
\kappa\left[\frac{\partial T}{\partial x}\right]=\frac{q\left(M_{0}-M_{b}\right) \varphi_{t}}{1+\varphi_{y}^{2}},
$$

where the square brackets denote a jump in a quantity across the reaction zone

$$
[v]=\left.v\right|_{x=0^{+}}-\left.v\right|_{x=0^{-}} .
$$

As the reaction zone is assumed to be very thin because of large activation energies, we can assume continuity of temperature across the front

$$
[T]=0 .
$$

From (A.1) and (A.2) we also have that

$$
\frac{A_{2}}{A_{1}} \frac{\partial J}{\partial x}=\frac{1}{M(1-\alpha M)} \frac{\partial M}{\partial x} .
$$

Integrating (A.8) from $x$ to the product end where $M=M_{b}$ and $J$ is essentially zero gives

$$
M=\frac{M_{b} \exp \left(\frac{J A_{2}}{A_{1}}\right)}{1-\alpha M_{b}+\alpha M_{b} \exp \left(\frac{J A_{2}}{A_{1}}\right)} .
$$

Evaluating (A.9) at the left end of the reaction zone where $M=M_{0}$ and $J=J_{0}$ yields

$$
M_{b}=\frac{M_{0} \exp \left(-j_{0}\right)}{1-\alpha M_{0}+\alpha M_{0} \exp \left(-j_{0}\right)}, \quad j_{0}=\frac{J_{0} A_{2}}{A_{1}} .
$$


Next, substituting (A.9) in (A.5) and using (A.10), we obtain

$$
\kappa \frac{\partial T}{\partial x}=-\frac{q \varphi_{t}}{1+\varphi_{y}^{2}} \quad \frac{M_{0} \exp \left(-j_{0}\right)}{1-\alpha M_{0}+\alpha M_{0} \exp \left(-j_{0}\right)} \frac{\exp \left(\frac{J A_{2}}{A_{1}}\right)}{1+\frac{\alpha M_{0}}{1-\alpha M_{0}} \exp \left(\frac{J A_{2}}{A_{1}}-j_{0}\right)} .
$$

Combining (A.11) with (A.1) yields

$$
\frac{\kappa}{\varphi_{t}} \frac{\partial T}{\partial x}=-\frac{q \varphi_{t}}{1+\varphi_{y}^{2}} \quad \frac{M_{0} \exp \left(-j_{0}\right)}{1-\alpha M_{0}+\alpha M_{0} \exp \left(-j_{0}\right)} \quad \frac{\frac{1}{J A_{1}} \exp \left(\frac{J A_{2}}{A_{1}}\right)}{1+\frac{\alpha M_{0}}{1-\alpha M_{0}} \exp \left(\frac{J A_{2}}{A_{1}}-j_{0}\right)} .
$$

Integrating this equation across the reaction zone, where $T$ varies from $T_{i}^{*}$ to $T_{b}$, and $J$ varies from $J_{0}$ to zero, yields the expression for $F\left(T_{b}\right)$.

\section{Appendix B: Coefficients}

The right-hand side of Equations (20-22) are

$$
\begin{aligned}
& \widetilde{Q}_{1}=0, \quad \widetilde{Q}_{2}=\frac{\partial}{\partial \tau_{1}}\left(\psi_{1} \frac{\mathrm{d} \widehat{\theta}}{\mathrm{d} \xi}-\theta_{1}\right)+\left(\frac{\partial \psi_{1}}{\partial \tau_{0}}-\frac{\partial^{2} \psi_{1}}{\partial \eta^{2}}\right) \frac{\partial \theta_{1}}{\partial \xi}+\frac{\partial \psi_{1}}{\partial \eta} \frac{\partial}{\partial \xi}\left(\frac{\partial \psi_{1}}{\partial \eta} \frac{\mathrm{d} \widehat{\theta}}{\mathrm{d} \xi}-2 \frac{\partial \theta_{1}}{\partial \eta}\right) \\
& \widetilde{Q}_{3}=\left(\frac{\partial \psi_{2}}{\partial \tau_{0}}-\frac{\partial^{2} \psi_{2}}{\partial \eta^{2}}\right) \frac{\partial \theta_{1}}{\partial \xi}+\frac{\partial}{\partial \tau_{1}}\left(\psi_{2} \frac{\mathrm{d} \widehat{\theta}}{\mathrm{d} \xi}-\theta_{2}\right)+\frac{\partial}{\partial \tau_{2}}\left(\psi_{1} \frac{\mathrm{d} \widehat{\theta}}{\mathrm{d} \xi}-\theta_{1}\right) \\
& +\frac{\partial \psi_{1}}{\partial \eta} \frac{\partial}{\partial \xi}\left(\frac{\partial \psi_{1}}{\partial \eta} \frac{\partial \theta_{1}}{\partial \xi}-2 \frac{\partial \theta_{2}}{\partial \eta}\right)+\frac{\partial \psi_{1}}{\partial \tau_{1}} \frac{\partial \theta_{1}}{\partial \xi} \\
& +2 \frac{\partial \psi_{2}}{\partial \eta} \frac{\partial}{\partial \eta}\left(\psi_{1} \frac{\mathrm{d}^{2} \widehat{\theta}}{\mathrm{d} \xi^{2}}-\frac{\partial \theta_{1}}{\partial \xi}\right)+\left(\frac{\partial \psi_{1}}{\partial \tau_{0}}-\frac{\partial^{2} \psi_{1}}{\partial \eta^{2}}\right) \frac{\partial \theta_{2}}{\partial \xi}, \\
& \widetilde{R}_{1}=0, \quad \widetilde{R}_{2}=\left(\frac{\partial \psi_{1}}{\partial \eta}\right)^{2}+\frac{\partial \psi_{1}}{\partial \tau_{1}}-m_{1 b} \frac{\partial \psi_{1}}{\partial \tau_{0}} \\
& \widetilde{R}_{3}=2 \frac{\partial \psi_{1}}{\partial \eta} \frac{\partial \psi_{2}}{\partial \eta}-\frac{\partial \psi_{1}}{\partial \tau_{0}}\left(\frac{\partial \psi_{1}}{\partial \eta}\right)^{2}+\frac{\partial \psi_{2}}{\partial \tau_{1}}+\frac{\partial \psi_{1}}{\partial \tau_{2}} \\
& -m_{1 b}\left(\left(\frac{\partial \psi_{1}}{\partial \eta}\right)^{2}+\frac{\partial \psi_{2}}{\partial \tau_{0}}+\frac{\partial \psi_{1}}{\partial \tau_{1}}\right)-m_{2 b} \frac{\partial \psi_{1}}{\partial \tau_{0}}, \\
& \widetilde{S}_{1}=0, \quad \widetilde{S}_{2}=-2 \frac{\partial \psi_{1}}{\partial \tau_{1}}-z_{2}\left(\theta_{1 b}\right)^{2}-\left(\frac{\partial \psi_{1}}{\partial \eta}\right)^{2}+\left(\frac{\partial \psi_{1}}{\partial \tau_{0}}\right)^{2} \\
& \widetilde{S}_{3}=-2 \frac{\partial \psi_{2}}{\partial \tau_{1}}-2 \frac{\partial \psi_{1}}{\partial \tau_{2}}+2 \frac{\partial \psi_{1}}{\partial \tau_{0}}\left(\frac{\partial \psi_{2}}{\partial \tau_{0}}+\frac{\partial \psi_{1}}{\partial \tau_{1}}\right)-2 z_{2} \theta_{1 b} \theta_{2 b} \\
& -z_{3}\left(\theta_{1 b}\right)^{3}-\mu \theta_{1 b}-z_{1 c r} \theta_{1 b}\left(\frac{\partial \psi_{1}}{\partial \eta}\right)^{2}-2 \frac{\partial \psi_{1}}{\partial \eta} \frac{\partial \psi_{2}}{\partial \eta}, \\
& \widetilde{T}_{1}=0, \quad \widetilde{T}_{2}=P_{2}\left(\theta_{1 b}\right)^{2}, \quad \widetilde{T}_{3}=2 P_{2} \theta_{1 b} \theta_{2 b}+P_{3}\left(\theta_{1 b}\right)^{3} .
\end{aligned}
$$


The coefficients $a_{\mathrm{i} j}, D_{\mathrm{i} j}$ and $C_{\mathrm{i}}$ appearing in the expressions for $g_{\mathrm{i}}(\xi)$ are

$$
\begin{aligned}
& D_{02}=-r_{0}, \quad D_{03}=\left(s^{2}+\mathrm{i} \omega\right) z_{1 c r}\left(\mathrm{i} \omega_{0}+z_{1 c r} / 2\right)(1-d)^{-1}, \\
& D_{04}=z_{1 c r}\left(s^{2}+\mathrm{i} \omega_{0}\right) \mathrm{i} \omega_{0}(1+d)^{-1}, \\
& D_{01}=-\left(1+P_{1}\right)^{-1}\left\{2 \omega_{0}^{2} P_{2}+s^{2} z_{1 c r}^{2} / 2+P_{1} \omega^{2} z_{1 c r}-\left((1-d) D_{04} / 2+C C\right)\right. \\
& \left.+\left((1+d) D_{03} / 2+C C\right)+P_{1}\left(D_{03}+C C\right)\right\}, \\
& a_{02}=D_{01}+D_{03}+\overline{D_{03}}-D_{04}-\overline{D_{04}} \\
& D_{13}=-\left(\mathrm{i} \omega_{0}+z_{1 c r} / 2\right)(1+d) z_{1 c r} / 4, \quad D_{14}=-\mathrm{i} \omega_{0} z_{1 c r}(1-d) / 4, \\
& a_{12}=\left\{D_{14}\left(\left(d+d_{1}\right) / 2+P_{1}\right)+D_{13}\left(d-d_{1}\right) / 2+z_{1 c r}^{2}\left(1-d_{1}\right) / 16\right. \\
& -\omega_{0}^{2} P_{2}-s^{2} z_{1 c r}^{2} / 4-P_{1} \omega_{0}^{2} z_{1 c r} / 2-\left(\omega_{0}^{2} z_{2}+s^{2} z_{1 c r}^{2} / 4\right. \\
& \left.\left.-z_{1 c r}^{2} \omega_{0}^{2} / 4-z_{1 c r} D_{14}\right)\left(4 \mathrm{i} \omega_{0}\right)^{-1}\left(0 \cdot 5-0 \cdot 5 d_{1}+2 \mathrm{i} \omega_{0}\right)\right\}\left\{\left(1-d_{1}\right) / 2\right. \\
& \left.-\left(1+z_{1 c r} / 4 \mathrm{i} \omega_{0}\right)\left(1+d_{1}\right) / 2+z_{1 c r} / 4 \mathrm{i} \omega_{0}+z_{1 c r} / 2-P_{1}\right\}^{-1} \\
& C_{1}=\left\{\omega_{0}^{2} z_{2}+s^{2} z_{1 c r}^{2} / 4-z_{1 c r}^{2} \omega_{0}^{2} / 4-z_{1 c r} a_{12}-z_{1 c r} D_{14}\right\}\left(4 \mathrm{i} \omega_{0}\right)^{-1}, \\
& D_{11}=C_{1}+z_{1 c r}^{2} / 8, a_{11}=a_{12}+D_{14}-D_{11}-D_{13} . \\
& D_{23}=-0 \cdot 5 z_{1 c r}(1+d)\left(\mathrm{i} \omega_{0}+0 \cdot 5 z_{1 c r}\right)=2 D_{13}, \\
& D_{24}=-0 \cdot 5 z_{1 c r} \mathrm{i} \omega_{0}(1-d)=2 D_{14}, \\
& a_{22}=\left\{0 \cdot 5\left(d-d_{2}\right) D_{23}+D_{24}\left\{0 \cdot 5\left(d+d_{2}\right)+P_{1}-z_{1}\left(4 \mathrm{i} \omega_{0}\right)^{-1}\left(1+2 \mathrm{i} \omega_{0}\right.\right.\right. \\
& \left.\left.-0 \cdot 5\left(1+d_{2}\right)\right)\right\}-z_{1 c r}^{2}\left(d_{2}-1\right) / 8+0 \cdot 5 s^{2} z_{1 c r}^{2}-P_{1} z_{1} \omega_{0}^{2}-2 P_{2} \omega_{0}^{2} \\
& \left.+\left(1+2 \mathrm{i} \omega_{0}-\left(1+d_{2}\right) / 2\right)\left(\omega_{0}^{2}\left(2 z_{2}-0 \cdot 5 z_{1 c r} 2\right)-0 \cdot 5 s^{2} z_{1 c r}^{2}\right)\left(4 \mathrm{i} \omega_{0}\right)^{-1}\right\} / \\
& \left\{-d_{2}+z_{1 c r}\left(1+2 \mathrm{i} \omega_{0}-0 \cdot 5\left(1+d_{2}\right)\right) / 4 \mathrm{i} \omega_{0}-P_{1}\right\} \\
& C_{2}=\left(-z_{1 c r} a_{22}-z_{1 c r} D_{24}-s^{2} z_{1 c r}^{2} / 2+2 \omega_{0}^{2} z_{2}-z_{1 c r}^{2} \omega_{0}^{2} / 2\right) / 4 i \omega_{0}, \\
& D_{21}=C_{2}+z_{1 c r}^{2} / 4, \quad D_{33}=D_{13}, \quad D_{34}=D_{14}, \\
& a_{21}=a_{22}\left(1+z_{1 c r} / 4 \mathrm{i} \omega_{0}\right)+D_{24}-z_{1 c r}^{2} / 4-D_{23} \\
& -\left(-z_{1 c r} D_{24}-s^{2} z_{1 c r}^{2} / 2+2 \omega_{0}^{2} z_{2}-z_{1 c r}^{2} \omega_{0}^{2} / 2\right) / 4 \mathrm{i} \omega_{0} \text {. } \\
& a_{32}=s^{2} z_{1 c r} / 2-2 \omega_{0}^{2} z_{2} / z_{1 c r}+z_{1 c r} \omega_{0}^{2} / 2-D_{34}-\overline{D_{34}},
\end{aligned}
$$




$$
\begin{aligned}
a_{31}= & 0 \cdot 5\left\{-a_{32}\left(\left(d_{3}+1\right) / 2+P_{1}\right)-\left(D_{34}\left((d+1) / 2-P_{1}\right)+C C\right)\right. \\
& \left.+\left(D_{33}(1-d) / 2+C C\right)+s^{2} z_{1 c r}^{2} / 2-2 \omega_{0}^{2} P_{2}-P_{1} \omega_{0}^{2} z_{1 c r}\right\}\left(d_{3}-1\right), \\
C_{3}= & a_{32}-a_{31}-z_{1 c r}^{2} / 4-D_{33}-\overline{D_{33}}+D_{34}+\overline{D_{34}}, \quad D_{31}=C_{3}+z_{1 c r}^{2} / 4 .
\end{aligned}
$$

The coefficients in the amplitude equations (24) are

$$
\begin{aligned}
& \chi=\mathrm{i} \omega_{0}\left(P_{1}+d\right)\left\{z_{1 c r}\left(P_{1}+d-z_{1 c r} / 2+2 \mathrm{i} \omega_{0} / d+z_{1 c r} /(2 d)\right)\right\}^{-1}, \\
& \beta_{1}=\left(Q_{5}-Q_{1}-Q_{3}\right)\left\{P_{1}+d-z_{1 c r} / 2+2 \mathrm{i} \omega_{0} / d+z_{1 c r} /(2 d)\right\}^{-1}, \\
& \beta_{2}=\left(Q_{6}-Q_{2}-Q_{4}\right)\left\{P_{1}+d-z_{1 c r} / 2+2 \mathrm{i} \omega_{0} / d+z_{1 c r} / 2 d\right\}^{-1}, \\
& Q_{1}+Q_{3}=2 \mathrm{i} \omega_{0} C_{1} I_{3}+r_{0} I_{2}-0.25 z_{1 c r}^{2} s^{2} I_{5}+0 \cdot 5 z_{1 c r}^{2} s^{2} I_{4}-2 z_{1 c r} s^{2} I_{7} \\
& +2 z_{1 c r} s^{2} C_{1} I_{1}+0 \cdot 5 z_{1 c r}\left(\mathrm{i} \omega_{0}+s^{2}\right) I_{6}+0 \cdot 5 z_{1 c r}\left(-\mathrm{i} \omega_{0}+s^{2}\right) I_{7}, \\
& Q_{2}+Q_{4}=2 \mathrm{i} \omega_{0} C_{2} I_{3}+r_{0} I_{2}+0 \cdot 5 z_{1 c r}^{2} s^{2} I_{5}-2 z_{1 c r} s^{2} I_{9}+2 z_{1 c r} s^{2} C_{3} I_{1} \\
& +0 \cdot 5 z_{1 c r}\left(\mathrm{i} \omega_{0}+s^{2}\right)\left(I_{6}+I_{9}\right)+0 \cdot 5 z_{1 c r}\left(-\mathrm{i} \omega_{0}+s^{2}\right) I_{7}, \\
& I_{1}=2 /(1+d) \\
& I_{2}=-\mathrm{i} \omega_{0} / d+0 \cdot 25 z_{1 c r}(d-1)\{1 / d-2 /(1+d)\}, \\
& I_{3}=2 \mathrm{i} \omega_{0} /(d+\bar{d})+0 \cdot 5 z_{1 c r}(d-1)\{1 /(d+\bar{d})-1 /(1+d)\}, \\
& I_{4}=-0 \cdot 5 \mathrm{i} \omega_{0}(d+1 / d)+1 / 8 z_{1 c r}(d-1)^{3} /\{d(d+1)\}-0 \cdot 25 z_{1 c r}(d-1), \\
& I_{5}=\mathrm{i} \omega_{0}(\bar{d}-d)+0 \cdot 25 z_{1 c r}(1-\bar{d})+\mathrm{i} \omega_{0}\left(d^{2}+1\right) /(d+\bar{d}) \\
& +0 \cdot 25 z_{1 c r}(d-1)^{2}\{1 /(d+1)-1 /(d+\bar{d})\}, \\
& I_{6}=0 \cdot 5(d+1)\left\{2 a_{02} /(d+1)+D_{04} / d+2 \overline{D_{04}} /(d+\bar{d})\right\} \\
& -(d-1)\left\{D_{01} /(d+1)-2 D_{02} /(d+1)^{2}+D_{03} /(2 d)+\overline{D_{03}} /(d+\bar{d})\right\}, \\
& I_{7}=-0 \cdot 5(d-1)\left\{2 a_{11} /\left(d+d_{1}\right)+2 D_{11} /(d+1)+D_{13} / d\right\} \\
& +0 \cdot 5(d+1)\left\{2 a_{12} /\left(d+d_{1}\right)+D_{14} / d\right\}, \\
& I_{8}=-0 \cdot 5(d-1)\left\{2 a_{21} /\left(d+d_{1}\right)+2 D_{21} /(d+1)+D_{23} / d\right\} \\
& +0 \cdot 5(d+1)\left\{2 a_{22} /\left(d+d_{1}\right)+D_{24} / d\right\},
\end{aligned}
$$




$$
\begin{aligned}
& I_{9}= 0 \cdot 5(d+1)\left\{2 a_{32} /\left(d+d_{3}\right)+D_{34} / d+2 \overline{D_{34}} /(d+\bar{d})\right\} \\
&-(d-1)\left\{a_{31} /\left(d+d_{3}\right)+D_{31} /(d+1)+D_{33} /(2 d)+\overline{D_{33}} /(d+\bar{d})\right\} \\
& Q_{5}=\left(P_{1}+d\right) / z_{1 c r}\left\{\mathrm{i} \omega_{0} z_{1 c r} r_{0}+2 \mathrm{i} z_{2} \omega_{0} g_{0}(0)+2 \omega_{0}^{2} z_{1 c r} C_{1}\right. \\
&\left.+3 \mathrm{i} \omega_{0} z_{1 c r}^{3} s^{2} / 4-2 s^{2} z_{1 c r} C_{1}+3 \mathrm{i} z_{3} \omega_{0}^{3}-2 \mathrm{i} z_{2} \omega_{0} g_{1}(0)\right\} \\
&-\mathrm{i}\left\{P _ { 1 } \omega _ { 0 } \left(-8 r_{0}+4 z_{1 c r} g_{0}(0)-4 z_{1 c r} g_{1}(0)\right.\right. \\
&\left.-6 s^{2} z_{1 c r}^{2}\right)+16 \mathrm{i} C_{1}\left(P_{1} \omega_{0}^{2}+s^{2} z_{1 c r}\right) \\
&\left.+3 \omega_{0} z_{1 c r}\left(s^{2} z_{1 c r}^{2}+4 \omega_{0}^{2} P_{2}\right)\right\} / 8 \\
&-2 \mathrm{i} P_{2} \omega_{0} g_{0}(0)+2 \mathrm{i} P_{2} \omega_{0} g_{1}(0)-3 \mathrm{i} P_{3} \omega_{0}^{3}, \\
& Q_{6}=\left(P_{1}+d\right) / z_{1 c r}\left\{2 \mathrm{i} \omega_{0} z_{2}\left(g_{3}(0)-g_{2}(0)+g_{0}(0)\right)+6 \mathrm{i} \omega_{0}^{3} z_{3}\right. \\
&\left.+2 \omega_{0}^{2} z_{1 c r} C_{2}+\mathrm{i} \omega_{0} z_{1 c r} r_{0}-2 s^{2} z_{1 c r} C_{3}-\mathrm{i} \omega_{0} z_{1 c r}^{3} s^{2} / 2\right\} \\
&-\mathrm{i}\left\{4 \omega_{0} z_{1 c r} P_{1}\left(g_{0}(0)-g_{2}(0)+g_{3}(0)+s^{2} z_{1 c r}\right)+16 s^{2} z_{1 c r} C_{3} P_{2}\left(g_{2}(0)-g_{0}(0)-g_{3}(0)\right)-6 \mathrm{i} P_{3} \omega_{0}^{3} .\right. \\
&\left.+24 \omega_{0}^{3} z_{1 c r} P_{2}-2 \omega_{0} z_{1 c r}^{3} s^{2}-8 P_{1} \omega_{0} r_{0}+16 \mathrm{i} P_{1} \omega_{0}^{2} C_{2}\right\} / 8 \\
&+
\end{aligned}
$$

\section{References}

1. N.M. Chechilo, R.J. Khvilivitskii and N.S. Enikolopyan, On the phenomenon of polymerization reaction spreading. Dokl. Akad. Nauk SSSR 204 (1972) 1180-1181.

2. J.A. Pojman, V.M. Ilyashenko and A.M. Khan, Spin mode instabilities in propagating fronts of polymerization. Physica D 84 (1995) 260-268.

3. D.A. Schult and V.A. Volpert, Linear stability analysis of thermal free radical polymerization waves. Int. J. Self-Propag. High-Temp. Synthesis 8 (1999) 417-440.

4. C.A. Spade and V.A. Volpert, Linear stability analysis of non-adiabatic free-radical polymerization waves. Combust. Theory Modelling 5 (2001) 21-39.

5. L.K. Gross and V.A. Volpert, Weakly nonlinear stability analysis of frontal polymerization. Stud. Appl. Math. 110 (2003) 351-375.

6. D.M.G. Comissiong, L.K. Gross and V.A. Volpert, Bifurcation analysis of polymerization fronts. In: J.A. Pojman and Q. Tran-Cong-Miyata (ed.), Nonlinear Dynamics in Polymeric Systems, number 869 in ACS Symposium Series, pp. 147-159. American Chemical Society, Washington, DC (2003).

7. G.G. Odian, Principles of Polymerization, 3rd ed. New York: Wiley-Interscience (1991) 768 pp.

8. C.A. Spade and V.A. Volpert, On the steady state approximation in thermal free radical frontal polymerization. Chem. Engng. Sci. 55 (2000) 641-654.

9. G.B. Manelis, L.P. Smirnov and N.I. Peregudov, Nonisothermal kinetics of polymerization processes: finite cylindrical reactor. Combust. Explos. Shock Wave 13 (1977) 389-393.

10. Ya.B. Zeldovich, G.I. Barenblatt, V.B. Librovich and G.M. Makhviladze, The Mathematical Theory of Combustion and Explosion. New York: Consultants Bureau (1985) 597 pp.

11. P.M. Goldfeder, V.A. Volpert, V.M. Ilyashenko, A.M. Khan, J.A. Pojman and S.E. Solovyov, Mathematical modeling of free-radical polymerization fronts. J. Phys. Chem. B 101 (1997) 3474-3482.

12. P.M. Goldfeder and V.A. Volpert, Nonadiabatic frontal polymerization. J. Engng. Math. 34 (1998) 301-318. 
13. M.F. Perry, V.A. Volpert, L.L. Lewis, H.A. Nichols and J.A. Pojman, Free-radical frontal copolymerization: the dependence of the front velocity on the monomer feed composition and reactivity ratios. Macromol. Theory Simul. 12 (2003) 276-286.

14. M.F. Perry and V.A. Volpert, Self-propagating free-radical binary frontal polymerization. J. Engng. Math. 49 (2004) 359-372.

15. A.G. Merzhanov and B.I. Khaikin, Theory of combustion waves in homogeneous media. Prog. Ener. Combust. Sci. 14 (1988) 1-98.

16. S.B. Margolis and B.J. Matkowsky, Flame propagation with a sequential reaction mechanism. SIAM J. Appl. Math. 42 (1982) 1175-1188.

17. V.A. Volpert and P.M. Krishenik, Nonsteady propagation of combustion waves in a system of successive reactions with endothermal stages. Combust. Explos. Shock Waves 22 (1986) 285-292.

18. C.A. Spade and V.A. Volpert, Mathematical modeling of interfacial gel polymerization for weak and strong gel effects. Macromol. Theory Simul. 9 (2000) 26-46.

19. H.G. Kaper, G.K. Leaf, S.B. Margolis and B.J. Matkowsky, Bifurcation of pulsating and spinning reaction fronts in condensed two-phase combustion. Combust. Sci. Technol. 43 (1985) 127-165.

20. S.B. Margolis and B.J. Matkowsky, Flame propagation in channels: secondary bifurcation to quasi-periodic pulsations. SIAM J. Appl. Math. 45 (1985) 93-129. 